\title{
Nephelometer Measurements of the Asymmetry Parameter, Volume Extinction Coefficient, and Backscatter Ratio in Arctic Clouds
}

\author{
H. GERBER \\ Gerber Scientific Inc., Reston, Virginia \\ Y. TAKANO \\ Department of Atmospheric Sciences, University of California, Los Angeles, Los Angeles, California \\ Timothy J. Garrett and Peter V. Hobbs \\ Department of Atmospheric Sciences, University of Washington, Seattle, Washington
}

(Manuscript received 16 July 1999, in final form 23 August 1999)

\begin{abstract}
A new instrument, a cloud integrating nephelometer (CIN), was used on the University of Washington's CV580 research aircraft to measure the asymmetry parameter $(g)$, volume scattering coefficient, and backscatter ratio in clouds in situ and in the visible spectrum during the FIRE-ACE/SHEBA field project in the late spring and summer of 1998 in the Arctic. The principle behind the operation of the CIN is described, and error estimates for the measurements are calculated. The average value of $g$ measured with the CIN in all the Arctic clouds was 0.824 , which is slightly less than the value expected for the water-containing clouds that dominated the measurements. The average value of $g$ measured in a glaciated cloud, containing mostly large bullet rosette ice crystals, was 0.737 . This relatively small value of $g$ is compared to modeled and indirect measurements of $g$ described in the literature, some of which gave similar small values of $g$.
\end{abstract}

\section{Introduction}

The asymmetry parameter, $g$, is a numerical value related to the difference between forward-scattered and backscattered electromagnetic radiation in dispersed systems, such as clouds and aerosols. Because of the computational inefficiency of using exact scattering phase functions, $g$ is often used to parameterize the phase function in the the two-stream radiation transfer approximation. Establishing the value of $g$ for atmospheric ice clouds has engendered a significant amount of recent interest, because the choice of $g$ strongly affects calculations of the radiative properties of ice clouds. For example, a large value of $g$ results in less reflected solar radiation from ice clouds than does a small value of $g$. The main theme of this paper is the measurement and calculation of $g$ for ice clouds. Measurements of the volume scattering and extinction coefficients and the backscatter ratio of ice crystal clouds are also discussed.

Corresponding author address: Dr. H. Gerber, Gerber Scientific Inc., 1643 Bentana Way, Reston, VA 20190.

E-mail: gerber.gsi@erols.com
Three basic ways are described in the literature for determining $g$ for ice crystals. The first entails using ray-tracing methods on model ice crystal geometries, such as hexagonal plates and columns, dendritic forms, and bullet rosettes (e.g., Takano and Jayaweera 1985; Takano and Liou 1989, 1995; Macke 1993; Mitchell et al. 1996). This method yields $g$ values in the visible spectrum ranging from about 0.750 to 0.940 depending on crystal habit and size. However, the models' idealized crystal shapes often do not precisely describe actual atmospheric ice crystals, which can exibit much greater complexity (Hobbs 1974). To account better for such complexity, various other ice crystal models have been hypothesized and $g$ calculated. Macke et al. (1996) use a many-faceted and nearly isometric polycrystal that gave a relatively small value of $g \approx 0.740$, and a similar value of $g$ is given by Mitchell et al. (1996) for planar polycrystals consisting of aggregates of the basic forms of ice crystals; Liou et al. (2000) describe regular aggregates with $g=0.752$ and irregular aggregates with $g=0.742$; and Macke and Mishchenko (1999) calculate $g$ for an ice crystal with multiple inclusions, such as small air bubbles, and obtain values of $g$ as small as about 0.550 for maximum inclusion density.

The second way to determine $g$ for ice clouds entails 
indirect measurements of $g$, where radiance fields are measured remotely from outside the cloud and then compared to the fields calculated from measured or assumed cloud structure. This approach has been used for ice cloud radiative measurements from satellites (Platt et al.1980; Wielicki et al. 1990), and from aircraft (Stephens et al. 1990; Stackhouse and Stephens 1991; Francis et al. 1994; Francis 1995; Mitchell et al. 1996; Macke et al. 1998). The earlier studies found $g \approx 0.700$, while the latter studies found $g$ generally greater than 0.800 . This difference can possibly be accounted for by different cloud geometry and microphysics, although some of these studies used the same clouds.

The final method of establishing $g$ is to measure it directly. Two such attempts, by Volkovitsky et al. (1979) and Sassen and Liou (1979a), entailed measurements of the angular distribution of scattered light (phase function) by ice crystal in a cloud chamber. Volkovitsky et al. (1979) found $g=0.850$ and Sassen and Liou (1979a) found $g=0.820$ (as calculated from their tabulated phase function). Francis (1995) pointed out that failure to measure light scattered in the near-forward direction in such direct measurements leads to a forward-scattering error that could cause considerable uncertainties in the derived values of $g$.

In summary, previous studies indicate that it is difficult to establish a representative value of $g$ for ice crystals and ice clouds, be it done by calculations for model ice crystals, by indirect atmospheric measurements, or by direct laboratory measurements.

We describe here a new approach for measuring $g$ directly and in clouds in the visible spectrum with a cloud integrating nephelometer (CIN), following the suggestions of Heintzenberg and Charlson (1996) and Gerber (1996a). The integrating nephelometer, first described by Beutell and Brewer (1949), optically integrates the phase function, which can also be achieved with a polar nephelometer by integrating measurements made at a large number of different scattering angles (e.g., see Gayet et al. 1997). In section 2 we describe the theory involved in adapting the integrating-nephelometer principle to the measurement of $g$. We show also that a measurement of $g$ requires a measure of the volume scattering coefficient $\left(\sigma_{s}\right)$, which is equivalent to the volume extinction coefficient $\left(\sigma_{e}\right)$ for nonabsorbing particles. We also describe means for partly correcting the forward-scattering error, which is an unavoidable part of measurements, that require integration of the scattering phase function over all scattering angles. Subsequent sections describe the CIN instrumentation, measurements of $g$ and $\sigma_{e}$ made from the University of Washington's CV-580 aircraft in ice and water clouds during the Arctic First International Satellite Cloud Climatology Project (ISCCP) Regional Experiment-Arctic Cloud Experiment/Surface Heat Budget of the Arctic Ocean Experiment (FIRE-ACE/SHEBA) in 1998, and conclusions.

\section{Theory}

The equation for $g$ can be written as (Twomey 1977)

$$
g=\frac{\int_{-1}^{1} \int_{r} \cos \theta\left(i_{1}+i_{2}\right) n(r) d r d \cos \theta}{\int_{-1}^{1} \int_{r}\left(i_{1}+i_{2}\right) n(r) d r d \cos \theta}
$$

where $\theta$ is the scattering angle with respect to the optical axis of the incident light, $i_{1}$ and $i_{2}$ are the scattering intensity functions, $r$ the particle radius, and $n(r)$ the size distribution of the particles.

Equation (1) shows that $g$ is given by the cosineweighted scattered light intensity integrated over all scattering angles divided by an expression equivalent to $\sigma_{s}$ times $4 \pi / \lambda^{2}$, where $\lambda$ is the wavelength of light. Expanding Eq. (1) gives

$$
g=\frac{\int_{0}^{\pi / 2} \int_{r} \cos \theta \sin \theta\left(i_{1}+i_{2}\right) n(r) d r d \theta-\int_{\pi / 2}^{\pi} \int_{r}|\cos \theta \sin \theta|\left(i_{1}+i_{2}\right) n(r) d r d \theta}{\int_{0}^{\pi} \int_{r} \sin \theta\left(i_{1}+i_{2}\right) n(r) d r d \theta},
$$

which shows that an in situ measurement of $g$ requires at least three sensors, given the two cosine-weighted integrals in the numerator and the denominator that is proportional to $\sigma_{s}$. The absolute sign in the second term of the numerator makes this integral positive permitting measurement of this quantity with a sensor.

Figure 1 shows a schematic of the configuration used with the CIN. Shown are two sensors that measure the forward-scattered light (S1) and backscattered light (S2) from particles in the collimated laser beam; their sum is the total scattered light $(T)$ given by the denominator in Eq. (2). The other two sensors also measure forwardscattered light (S3) and backscattered light (S4), but these signals are now weighted with a "cosine mask" that produces the required "cosine-weighting" in the two terms of the numerator of Eq. (2). The sensors must 


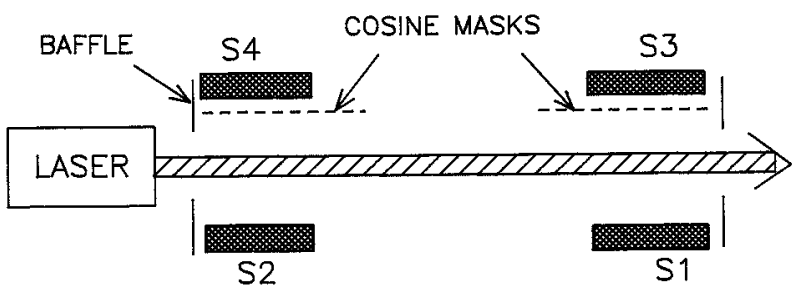

FIG. 1. Side view schematic sketch (not to scale) of the CIN. A laser beam irradiates airborne particles that scatter light into four sensors (S1-S4) with Lambertian responses. The S1 and S3 measure forward-scattered light; S2 and S4 measure backscattered light; and the measurements by S3 and S4 are weighted by the cosine of the scattering angle using a cosine mask. Each sensor consists of a circular light-diffusing disk and a photomultiplier.

have a Lambertian response over the angular scattering ranges indicated in Eq. (2). Without the mask and baffles, the combination of a Lambertian sensor and the light beam forms a configuration equivalent to one version of the integrating nephelometer described by Beutell and Brewer (1949) that gives a direct measure proportional to $\sigma_{s}$ of the particles irradiated by the light beam. The behavior of this type of nephelometer has been described by Gerber (1982).

Equation (1) shows that the range of integration of $\theta$ is $0 \leq \theta \leq \pi$, which is impossible to achieve from a measurement standpoint, because as $\theta$ approaches the limits, interference between the scattered light and the light beam cannot be avoided. This problem is especially significant for the lower limit, because there is a peak in forward scattering from particles. To judge the severity of this effect for the CIN, the error in $g$ is calculated in Fig. 2 from phase functions for a typical ice crystal and water droplet as a function of the lower integration limit $\theta_{1}$, below which a baffle would prevent scattered light from reaching sensors S1 and S3. The ice crystal " $\delta$-transmission" peak for $\theta=0^{\circ}$ (Takano and Liou 1989) is not included in this calculation and will be discussed below. Figure 2 shows that to reduce the error in the measured value of $g$ to less than about $5 \%$, the value of $\theta_{1}$ must be $<0.2^{\circ}$ for the crystal and $<1^{\circ}$ for the droplet. Neither of these angles is practical; integrating nephelometers used in the field have $\theta_{1}$ on the order of $6^{\circ}$ and larger (Rabinoff and Herman 1973).

The following correction scheme is applied to the measurements made by the four sensors to permit use of a practical value of $\theta_{1}$ : Mie theory predicts that light scattered by nonaborbing particles consists of the sum of diffracted light $(D)$ and of refracted and reflected light $(R)$, with $D$ for particles with $r \gg \lambda$ being concentrated in a range of small $\theta$ that nephelometers have difficulty measuring. The theory also predicts that $D=$ $R$ for large spheres and $D \approx R$ for ice crystals (Takano and Liou 1989). These predictions raise the possibility that a measure of $R$ that is more equally spread over the entire range of $\theta$ than is $D$ can be used first to estimate $D$ and then to estimate the total scattered light $T$ and $g$. To test this possibility, we will assume that an angle $\theta_{1}$ exits where the contributions of $D$ and $R$ can be separated so that the fraction of light $f$ scattered by diffraction is given by

$$
f=\frac{\int_{0}^{\theta_{1}} \int_{r} \sin \theta\left(i_{1}+i_{2}\right) n(r) d r d \theta}{\int_{0}^{\pi} \int_{r} \sin \theta\left(i_{1}+i_{2}\right) n(r) d r d \theta} .
$$

Here, $D=f \times T ; T=D+R ; R=\mathrm{R} 1+\mathrm{R} 2$; and the cosine-weighted forward-scattered and backscattered light are R3 $+D$ and $\mathrm{R} 4$, respectively, where R1, R2, $\mathrm{R} 3$, and R4 are the refracted and reflected light components measured by the respective sensors.

Applying the foregoing to Eq. (2) yields

$$
g=\frac{\int_{\theta_{1}}^{\pi / 2} \int_{r} \cos \theta \sin \theta\left(i_{1}+i_{2}\right) n(r) d r d \theta+D-\int_{\pi / 2}^{\theta_{2}} \int_{r}|\cos \theta \sin \theta|\left(i_{1}+i_{2}\right) n(r) d r d \theta}{\int_{\theta_{1}}^{\theta_{2}} \int_{r} \sin \theta\left(i_{1}+i_{2}\right) n(r) d r d \theta+D} \text { or }
$$

$$
\begin{aligned}
& g=\frac{\mathrm{R} 3+D-\mathrm{R} 4}{\mathrm{R} 1+D+\mathrm{R} 2} \text { or } \\
& g=\frac{(\mathrm{R} 1+\mathrm{R} 2) f+(\mathrm{R} 3-\mathrm{R} 4)(1-f)}{\mathrm{R} 1+\mathrm{R} 2},
\end{aligned}
$$

where the upper truncation angle $\theta_{2}=175^{\circ}$ has been added and $D$ has been eliminated from the desired result shown in Eq. (6).

The assumption used in writing Eq. (3) is tested by calculating $f$ for water droplets with a wide range of normal size distributions described by a mean droplet radius $\bar{R}$ and standard deviation $\sigma$, as shown in Fig. 3 . The distribution with $\bar{R}=10 \mu \mathrm{m}$ closely resembles that found in nonprecipitating Sc (Gerber 1996b). Figure 3 illustrates that it is not possible to find a value of $\theta_{1}$ where $f=0.5$ for all droplet size distributions; however, it shows that for distributions with the largest droplets $f \approx 0.5$ for small values of $\theta_{1}$. These results are consistent with Mie theory, which also predicts that both 


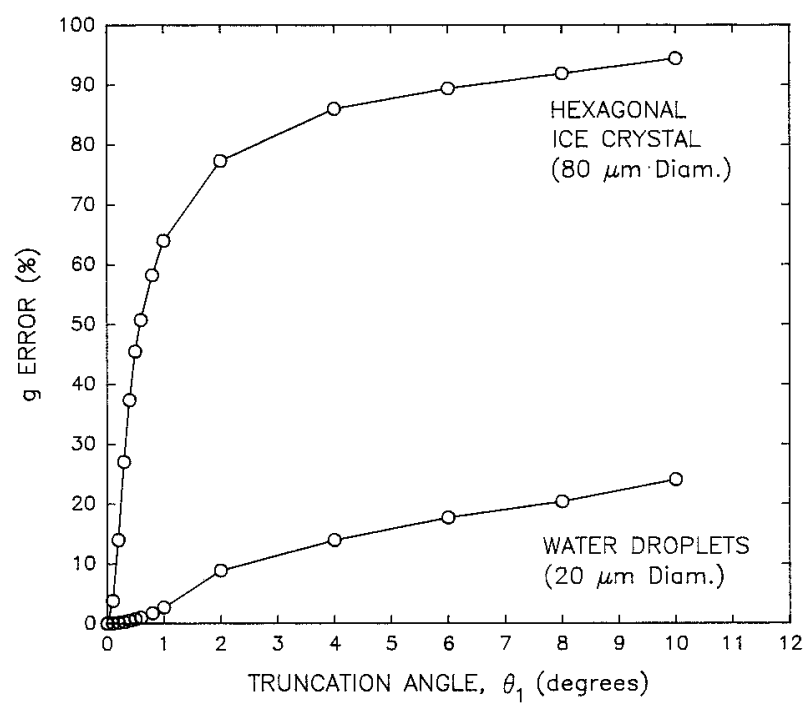

FIG. 2. Calculated error in the measurement of the asymmetry parameter $g$ for a typical ice crystal and water droplet as a function of the forward truncation angle $\theta_{1}$ of an integrating nephelometer.

$D$ and $R$ extend over the entire range of $\theta$. Thus, the assumption used for Eq. (3) includes an approximation that generates errors that affect the value of $f$ in Eq. (6). Figure 3 shows that for $\theta$ greater than about $6^{\circ}$ the variability of $f$ caused by different values $\bar{R}$ decreases, so that a practical choice of $\theta_{1}$ should come from $\theta>$ $6^{\circ}$. The choice made for the CIN is $\theta_{1}=10^{\circ}$, as indicated by the data point in Fig. 3. The range in $f$ is relatively small at that value of $\theta_{1}$ and reaches a nearly constant value for the largest droplets. The calculations of Fig. 3 were repeated with a similar range of droplet distributions given by $\sigma=0.40 \bar{R}$, and results closely resembling those in Fig. 3 were found.

Values of $f$ shown in Fig. 4 are calculated from phase functions at $\lambda=0.55 \mu \mathrm{m}$ derived by ray tracing for a variety of individual ice crystal shapes (Takano and Liou 1989, 1995), and from average phase functions based on a distribution of different ice crystal shapes found in ice clouds (Liou 1992). The value of $f$ at $\theta_{1}=10^{\circ}$ in Fig. 4 has more variability than for the droplets show in Fig. 3 and is somewhat larger on the average (see Table 1).

The error in $g$ in Eq. (6) caused by the variability of $f$ shown in Figs. 3 and 4, as well as by the choice of $\theta_{2}$, can be estimated by comparing the exact expression for $g$ [Eq. (2)] with Eq. (6), which describes $g$ measured by the CIN. The value of $f$ used with Eq. (6) is 0.54, which is estimated to be a median value for droplets and ice crystals, and which should be used when the composition of the cloud is unknown. The results of this comparison are shown in Fig. 5 with 2\% error limits and are summarized in Table 2. Table 2 shows that the estimated error in $g$ depends on additional information available about the cloud particles. For example, if the cloud is known to be composed only of ice crystals, a

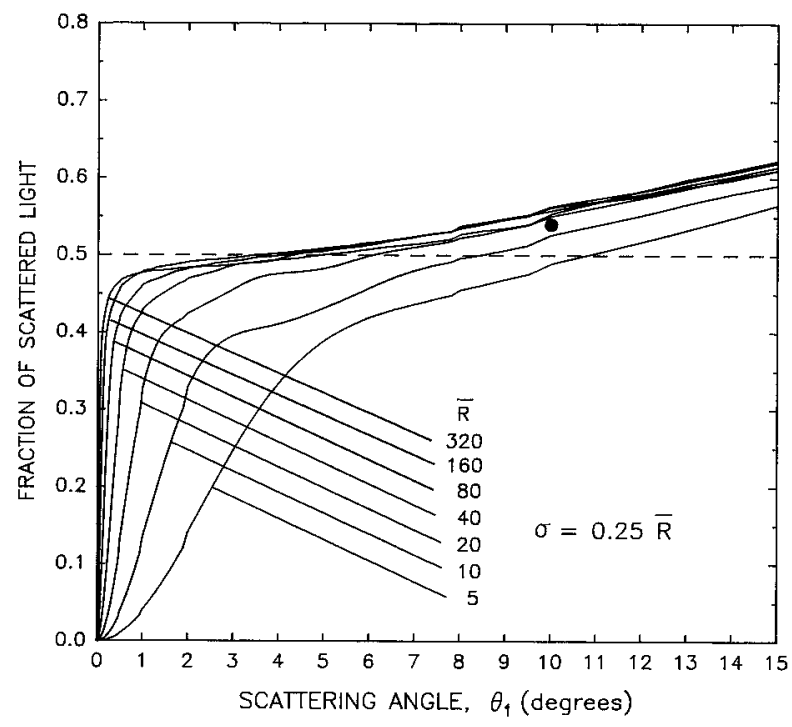

FIG. 3. Fraction of scattered light $f$ [Eq. (3)] as a function the scattering angle $\theta_{1}$ for normally distributed droplet size distributions with the given standard deviation $\sigma$ and mean droplet radius $\bar{R}$.

value of $f=0.57$ is a better choice for use with Eq. (6), and a value of $f=0.52$ is a better choice when the cloud contains only water. Figure 5 and Table 2 suggest that the natural range in $g$ is sufficient, and the error in its measurement with a perfect CIN is small enough so that meaningful measurements of $g$ used with a diffraction correction based on phase functions can be made.

The results of a similar error analysis are shown in Table 2 for $\sigma_{e}$, which is measured by the sum of $\mathrm{S} 1$ and S2 and is given by

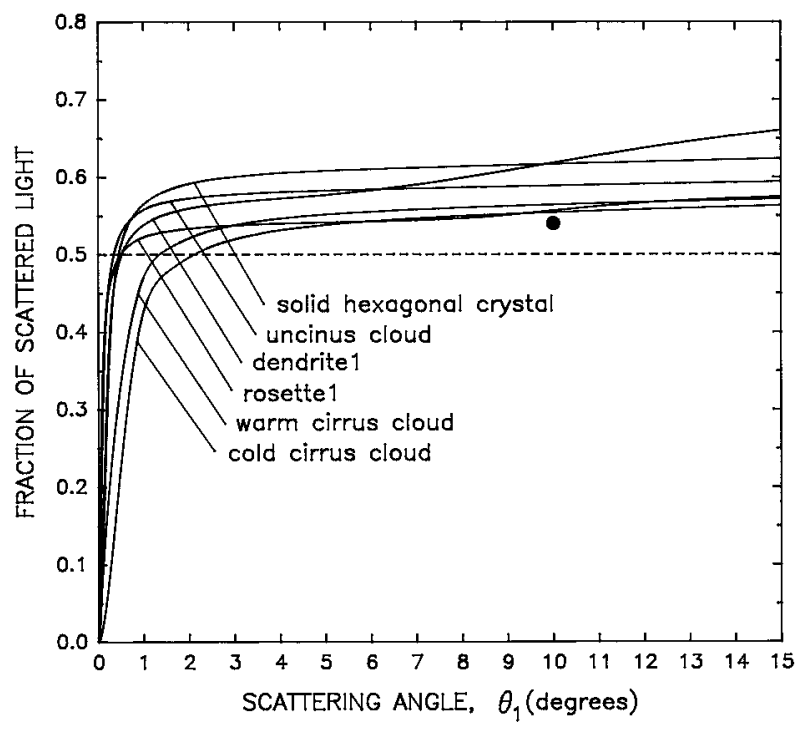

FIG. 4. Fraction of scattered light $f$ [Eq. (3)] as a function of the scattering angle $\theta_{1}$ based on phase functions calculated for ice clouds and ice crystals by Takano and Liou $(1989,1995)$ and Liou (1992). See Table 2. 
TABLE 1. Properties for different types of ice crystals and ice crystal clouds. The fractional scatter $f$ is derived from Eq. (3); $g$ is the asymmetry parameter based on scattered-light phase function from Eq. (2) and Eq. (6); $f_{\delta}$ is the fraction of $\delta$ transmission; $g_{i}$ is the assymmetry parameter of the ice crystals from Eq. (8); and $g_{i}-g$ is the difference between Eq. (8) and Eq. (2). The four cirrus clouds are described by Liou (1992); the definition for the ice crystal dimensions is given by Takano and Liou (1995); and the aggregates are described by Liou et al. (2000).

\begin{tabular}{|c|c|c|c|c|c|c|c|c|}
\hline No. & Type & $\begin{array}{l}\text { Dimensions } \\
\qquad(\mu \mathrm{m})\end{array}$ & $\begin{array}{c}f \\
\text { Eq. (3) }\end{array}$ & $\begin{array}{c}g \\
\text { Eq. (2) }\end{array}$ & $\begin{array}{c}g \\
\text { Eq. (6) }\end{array}$ & $f_{\delta}$ & $\begin{array}{c}g_{i} \\
\text { Eq. }(8)\end{array}$ & $\begin{array}{c}g_{i}-g \\
\text { Eqs. (8) }- \\
\text { (2) }\end{array}$ \\
\hline 1 & Cold cirrus & & 0.5618 & 0.7399 & 0.7340 & 0.1216 & 0.7715 & 0.0316 \\
\hline 2 & Warm cirrus & & 0.5695 & 0.7560 & 0.7459 & 0.1290 & 0.7874 & 0.0315 \\
\hline 3 & Cirrostratus & & 0.5572 & 0.7510 & 0.7426 & 0.1260 & 0.7824 & 0.0314 \\
\hline 4 & Uncinus cirrus & & 0.5900 & 0.8067 & 0.7886 & 0.1545 & 0.8366 & 0.0299 \\
\hline 5 & Solid hexagon & $L / 2 a=32 / 80$ & 0.6185 & 0.7694 & 0.7296 & 0.1931 & 0.8139 & 0.0445 \\
\hline 6 & Dendrite 1 & $\begin{aligned} L / 2 a & =32 / 80, \\
\mathrm{bb} & =8, \mathrm{bt}=20\end{aligned}$ & 0.6203 & 0.7995 & 0.7678 & 0.1368 & 0.8269 & 0.0274 \\
\hline 7 & Dendrite 2 & $\begin{aligned} L / 2 a & =32 / 80, \\
\mathrm{bb} & =12, \mathrm{bt}=40\end{aligned}$ & 0.6444 & 0.8207 & 0.7886 & 0.1192 & 0.8421 & 0.0214 \\
\hline 8 & Hollow column 1 & $\begin{array}{l}L / 2 a=300 / 100 \\
\quad \mathrm{dbar}=15\end{array}$ & 0.5877 & 0.8201 & 0.8039 & 0.1399 & 0.8453 & 0.0252 \\
\hline 9 & Hollow column 2 & $\begin{array}{l}L / 2 a=300 / 100 \\
\text { dbar }=75\end{array}$ & 0.5753 & 0.8424 & 0.8344 & 0.0944 & 0.8573 & 0.0149 \\
\hline 10 & Bullet rosette 1 & $\begin{array}{l}L / 2 a=120 / 60 \\
\quad t=48, \text { delta }=10\end{array}$ & 0.5581 & 0.7657 & 0.7634 & 0.0842 & 0.7854 & 0.0197 \\
\hline 11 & Bullet rosette 2 & $\begin{array}{l}L / 2 a=240 / 80, \\
\quad t=120, \text { delta }=20\end{array}$ & 0.5718 & 0.8140 & 0.8050 & 0.0842 & 0.8297 & 0.0157 \\
\hline 12 & Bullet rosette 3 & $\begin{array}{l}L / 2 a=120 / 48 \\
\quad t=48, \text { delta }=10\end{array}$ & 0.5457 & 0.7826 & 0.7856 & 0.0928 & 0.8028 & 0.0202 \\
\hline 13 & Regular aggregates & & 0.5547 & 0.7241 & 0.7215 & 0.1001 & 0.7517 & 0.0276 \\
\hline 14 & Irregular aggregates & & 0.5892 & 0.7413 & 0.7188 & 0.0038 & 0.7423 & 0.0010 \\
\hline
\end{tabular}

$$
\sigma_{e}=\sigma_{s}=C \frac{\mathrm{R} 1+\mathrm{R} 2}{1-f},
$$

where $C$ is a scaling constant. Here and in the following we assume that the droplets and ice crystals do not

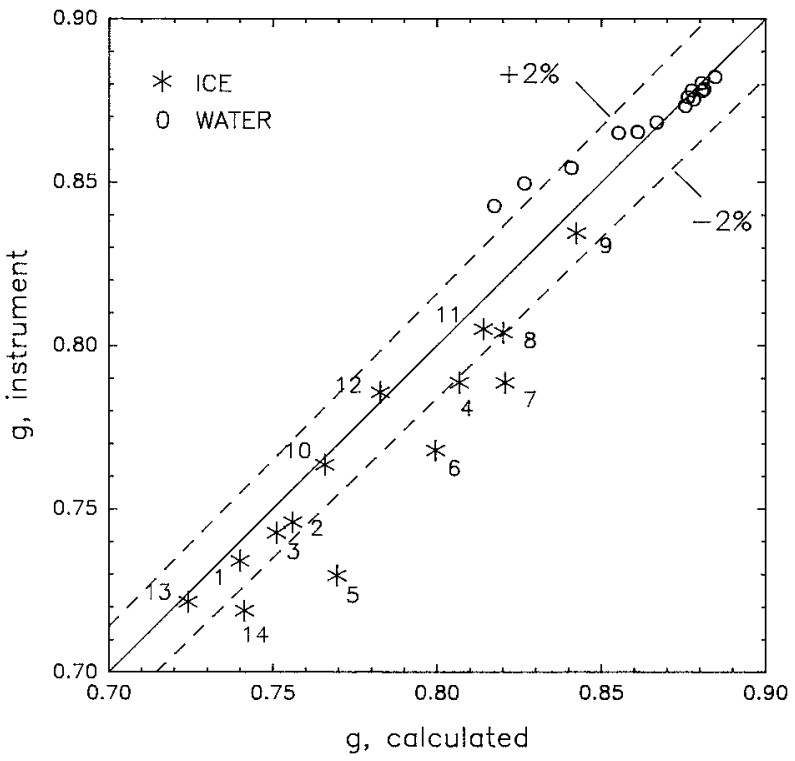

FIG. 5. Comparison between $g$ calculated with the exact expression [Eq. (2)] and $g$ for an integrating nephelometer for which truncation angles cause errors that are partly corrected with a value of $f=0.54$ [Eq. (6)]. Error intervals of $2 \%$ are shown. The numbers refer to the ice crystal type listed in Table 1. absorb any light, so that $\sigma_{e}=\sigma_{s}$. It is important to note that $\sigma_{e}$ as well as $g$ measured in this fashion have a mimimal dependence on the hydrometeror size, provided the size is larger than about 5- $\mu \mathrm{m}$ diameter (as illustrated in Fig. 3).

Up to this point the determination of $g$ for ice crystals has been related only to their phase functions. It is also necessary to take into account the effect of the $\delta$-function transmission through ice crystals on the value of $g$. The transmission occurs at $\theta=0^{\circ}$ when opposing facets of crystals are exactly parallel so that some light passes through the crystal without deviation. This effect on $g$ is given by Takano and Liou (1989) as

$$
g_{i}=\left(1-f_{\delta}\right) g+f_{\delta},
$$

where the corrected value of $g$ for ice crystals is $g_{i}$, which now depends on the fraction $f_{\delta}$ of light transmitted at $\theta=0^{\circ}$ to the total scattered light. Values of $f_{\delta}$ based on calculations by Takano and Liou (1989, 1995), Liou (1992), and Liou et al. (2000) are listed in Table 1. The effect of $f_{\delta}$ is to increase the value of $g$.

TABLE 2. Estimates of measurement error in percent for the CIN vs information known about the cloud.

\begin{tabular}{llc}
\hline \hline \multicolumn{1}{c}{ Information } & $g(\%)$ & $\sigma_{e}(\%)$ \\
\hline Cloud composition unknown & \pm 3 & \pm 15 \\
Ice crystals & \pm 2 & \pm 7.5 \\
Water droplets & \pm 1.5 & \pm 7.5 \\
Crystal habits known & \pm 1 & \pm 2.5 \\
Relative droplet spectrum known & \pm 0.5 & \pm 1 \\
\hline
\end{tabular}




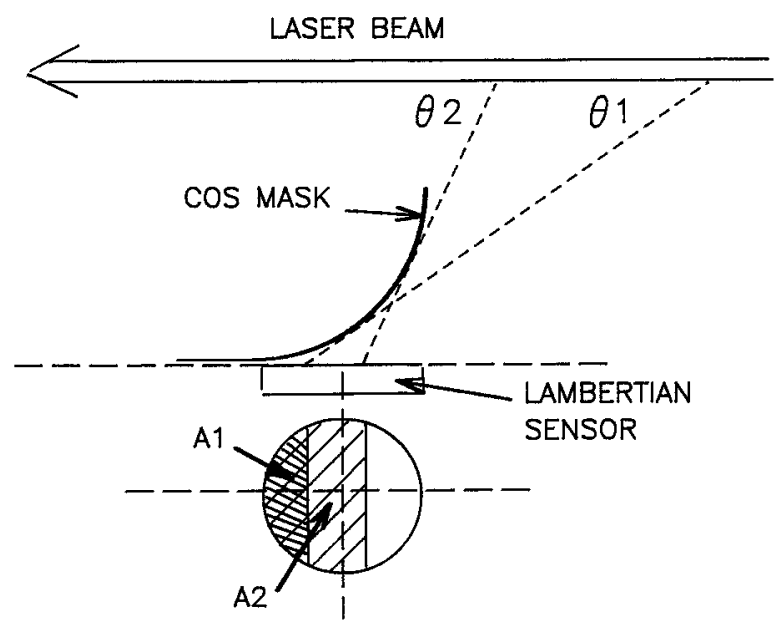

FIG. 6. Side and top view of the circular Lambertian diffuser shown with the location of the cosine mask. The mask shadows the surface of the diffuser so that the area " $\mathrm{A}$ " shadowed by the mask is proportional to $1-|\cos \theta|$, where $\theta$ is the scattering angle.

Table 1 shows that the difference $g_{i}-g$ [Eq. (6) - Eq. (2)] is largest for solid hexagonal ice crystals and has a nearly constant value of 0.03 for the four different and typical ice clouds. The $\delta$-function transmission has not been measured for atmospheric ice crystals; thus it constitutes an additional source of uncertainty in the measurement of $g_{i}$.

The ratio of the backscattered light to total scattered light is the backscatter ratio of the ice crystals and is given by

$$
b_{r}=\frac{\mathrm{R} 2(1-f)}{(\mathrm{R} 1+\mathrm{R} 2)\left(1+f_{\delta}\right)} .
$$

\section{Design of the CIN}

The sensors of the CIN, which are sketched in Figs. 1 and 6, consist of a combination of circular plastic disks that act as Lambertian diffusers and photomultipliers that measure the light transmitted throught the disks. Each pair of sensors on either side of the laser beam is mounted in a wing-shaped arm, each of which resembles half of a strut with an elliptical cross section that is split parallel to its long axis to form each wing. The diffusers and masks are mounted on the inside flat surface of the wings, which are placed parallel to each other and $3.5 \mathrm{~cm}$ apart. The laser beam is projected down the middle. The CIN is mounted on the aircraft so that the long dimension of the arms is perpendicular to the wind direction and the flat surfaces are parallel to the wind direction, which minimizes deviations of the streamlines through the $3.5-\mathrm{cm}$ gap.

The volume of atmosphere irradiated by the laser beam and seen by the sensors is about $30 \mathrm{~cm}^{3}$, which gives a volume sampling rate of $300 \mathrm{~L} \mathrm{~s}^{-1}$ at an aircraft speed of $100 \mathrm{~m} \mathrm{~s}^{-1}$. The optical measurements are in-

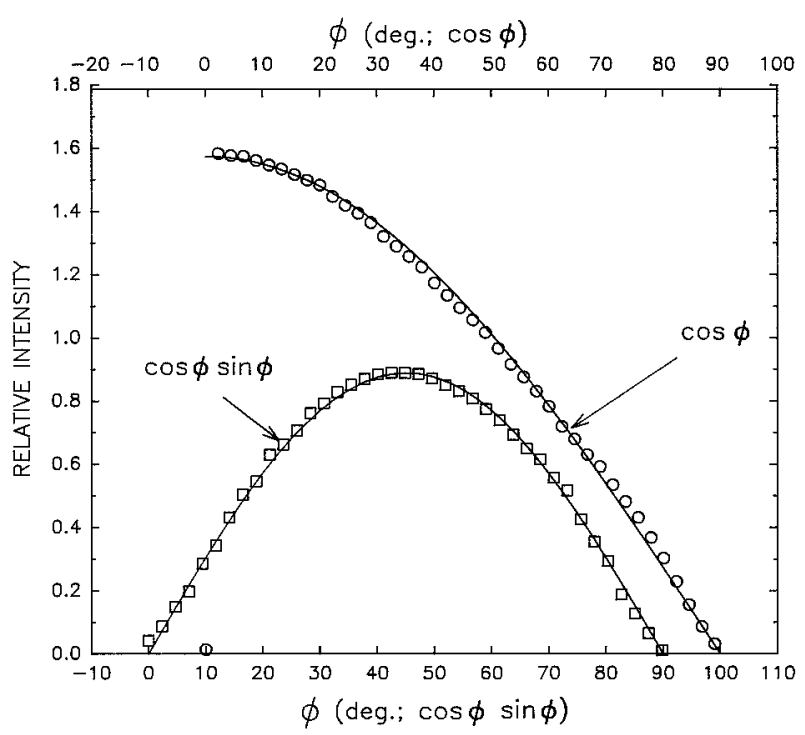

FIG. 7. Comparison of the measured relative responses (data points) of a CIN sensor with and without the cosine mask to the required exact response (curves). The angle $\phi$ is measured from the normal to the surface of the circular diffusing disk, and is the complement of the scattering angle $\theta$. The upper and lower scales are shifted $10^{\circ}$ for clarity.

dependent of airspeed, and the laser has a wavelength of $635 \mathrm{~nm}$.

A crucial design feature of the CIN is the accurate performance of the Lambertian diffusers and the cosine masks. The design of the masks is illustrated schematically in Fig. 6. The mask consists of a baffle with a circular cross section placed between the diffuser and the laser beam. It can be shown that a size and position of the mask can be chosen so that the area " $A$ " it shadows from the scattered laser light is proportional to 1 $-|\cos \theta|$. Figure 7 compares the ideal behavior of the diffusers with and without the cosine mask to laboratory response measurements; the angle $\phi$ is the complement of $\theta$ and is measured from the normal to the circular diffuser disks.

The measurement accuracy also depends on the sensitivity of each photomultiplier associated with each diffuser. Given that the measure of $g$ and $b_{r}$ are relative measurements, it is necessary to match the sensitivity of all photomultipliers. This is done by removing all baffles and masts, and simultaneously exposing all sensors to a point source of light placed at a distance from the sensors.

To measure $\sigma_{e}$ it is also necessary to compare the sensitivity of the sensors with an instrument of known sensitivity to establish the scaling constant $C$ in Eq. (7). The ideal method would be to compare the CIN with a transmissometer operating in a cloud. This has not been done. Instead the CIN was compared to a particle volume monitor (PVM; Gerber 1991) which has the capability of measuring the [total particle surface area 


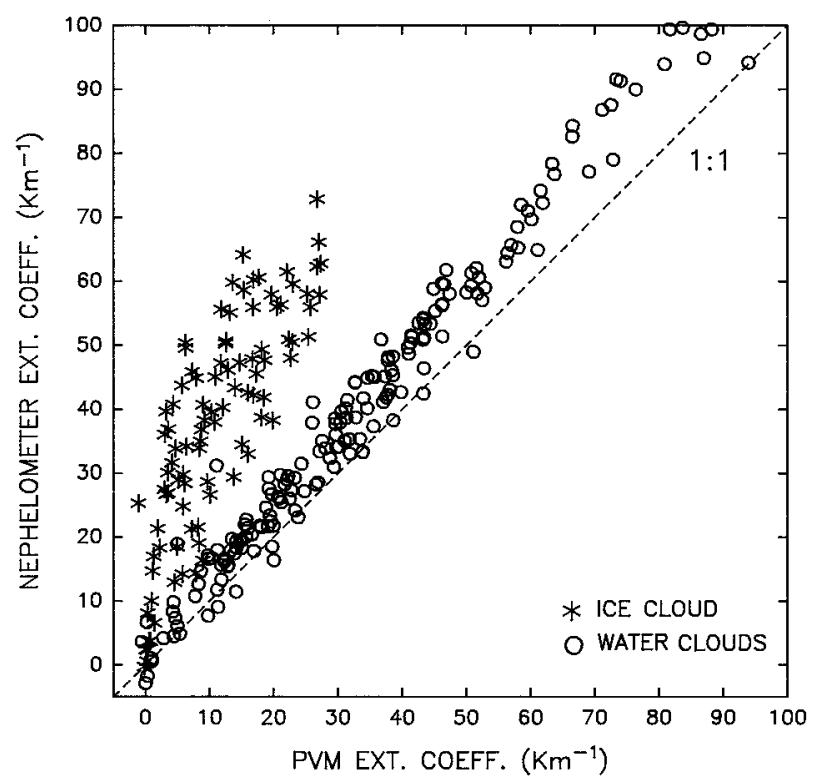

FIG. 8. Comparison of the volume extinction coefficient measured by the CIN and PVM on the CV-580 aircraft during the FIRE-ACE/ SHEBA experiment for some ice and water clouds. The coefficients for the ice cloud were multipled by a factor of 2 to permit the difference between CIN and PVM measurements to be seen more easily.

(PSA; $\left.\mathrm{cm}^{2}\right)$ per volume $\left(\mathrm{m}^{-3}\right)$ of atmosphere]. PSA and $\sigma_{e}$ are related as follows: The definition of $\sigma_{e}$ is

$$
\sigma_{e}=\int_{0}^{\infty} \pi r^{2} Q_{e} n(r) d r,
$$

where $Q_{e}$ is the efficiency factor for extinction. For droplets with $\mathrm{r} \gg \lambda, Q_{e} \approx 2.0$ so that

$$
\begin{aligned}
\sigma_{e} & \approx \frac{1}{2} \int_{0}^{\infty} 4 \pi r^{2} n(r) d r \\
& =0.05 \text { PSA km }{ }^{-1} \mathrm{~cm}^{-2} \mathrm{~m}^{3},
\end{aligned}
$$

which is combined with Eq. (7) to give

$$
C=\frac{0.05(1-f) \mathrm{PSA}}{\mathrm{R} 1+\mathrm{R} 2} V^{-1} \mathrm{~km}^{-1},
$$

where $V$ is the voltage outputs measured for R1 and R2.

The CIN and a PVM were compared in a cloud chamber prior to the FIRE-ACE/SHEBA field study to establish a value of $C$, and the CIN was compared to another PVM located on the University of Washington CV-580 aircraft during the experiment. Figure 8 shows the latter comparison where the CIN $\sigma_{e}$ is given by Eq. (7) and the PVM $\sigma_{e}$ is given by Eq. (11). The water cloud data, taken on 20 May 1998, represent aircraft profiles through five layer clouds containing only water droplets. The ice cloud data was taken on 1 June 1998 in a glaciated cloud at a temperature of $-42^{\circ} \mathrm{C}$ that contained primarily bullet rosettes with a mean diameter of about $150 \mu \mathrm{m}$. The expected high correlation between CIN and PVM $\sigma_{e}$ measurements for the water clouds was found. However, the scaling between the two probes is off by about $15 \%$. This difference may be related to the uncertainty in the calibration constants for the PVM and in the assumption that $Q_{e}=2.00$ in Eq. (11); $Q_{e}$ actually varies from about 1.95 to 2.30 for the small droplets typical of those used in the cloud chamber comparison and those found in the layer clouds.

The large difference between $\sigma_{e}$ measured by the CIN and the PVM for the ice cloud illustrates that the PVM, with a built-in upper-size roll-off starting for particles of about $40-\mu \mathrm{m}$ diameter, strongly underestimates $\sigma_{e}$ for these large ice crystals, whereas the CIN measures $\sigma_{e}$ more accurately, because it does not have such an upper-size limit.

\section{Measurements}

\section{a. Water cloud}

The CIN was mounted pointing downward on the underside of the fuselage and just behind the nose wheel of the CV-580 aircraft. The aircraft flew out of Barrow, Alaska, and was used to measure cloud properties in the Barrow area, over the Barrow atmospheric radiation measurements (ARM) site, and on several flights to the SHEBA ship, which was several hundred kilometers NW of Barrow.

Figures 9-11 illustrate 1-Hz CIN measurements made on 20 May 1998 during a horizontal traverse of a segment of stratocumulus cloud located in the vicinity of the ARM site. This cloud contained water droplets, as shown in Fig. 9 by the images collected by the cloud particle imager (CPI; Lawson 1997). The outputs of the four channels of the CIN are shown in Fig. 10, where $\mathrm{R} 1, \mathrm{R} 2, \mathrm{R} 3$, and R4 are now defined more descriptively as forward-scattered light $(F)$, backscattered light $(B)$, cosine-weighted forward-scattered light $(\cos F)$; and cosine-weighted backscattered light $(\cos B)$, respectively. Figure 10 shows that $F$ and $\cos F$ are much larger than $B$ and $\cos B$. The average backscatter ratio $b_{r}$ for this cloud is 0.036 .

The average value of $g$ tabulated in the FIRE-ACE data archive (Distributed Active Archive Center, located at National Aeronautics and Space Administration Langley Research Center) for the 5-min increment of the stratocumulus cloud shown in Fig. 11 is 0.844 . This value of $g$ corresponds to the use of $f=0.530$ with Eq. (6), which is applied to all the archived data. The droplet spectrum in this cloud has a relatively small measured value of $6.0 \mu \mathrm{m}$ for the droplet effective radius $\left(R_{e}\right)$, which corresponds better to a value of $f \approx$ 0.50 . Given that the sensitivity of $g$ to a change in $f$ for the water droplets of that size is found to be $\Delta g / \Delta f$ $\approx 0.30$, the approximate average value of $g$ for the 5-min cloud increment must be reduced to 0.835 (standard deviation 0.025) as shown in Fig. 11. This value of $g$ is close to the value calculated with the exact expession for $g$ given by Eq. (2), suggesting that the CIN 
201199ิ

4U:19: 14

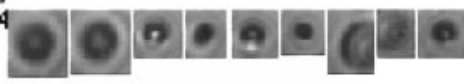

2J:40:19: 5

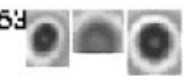

21:40:19: 8
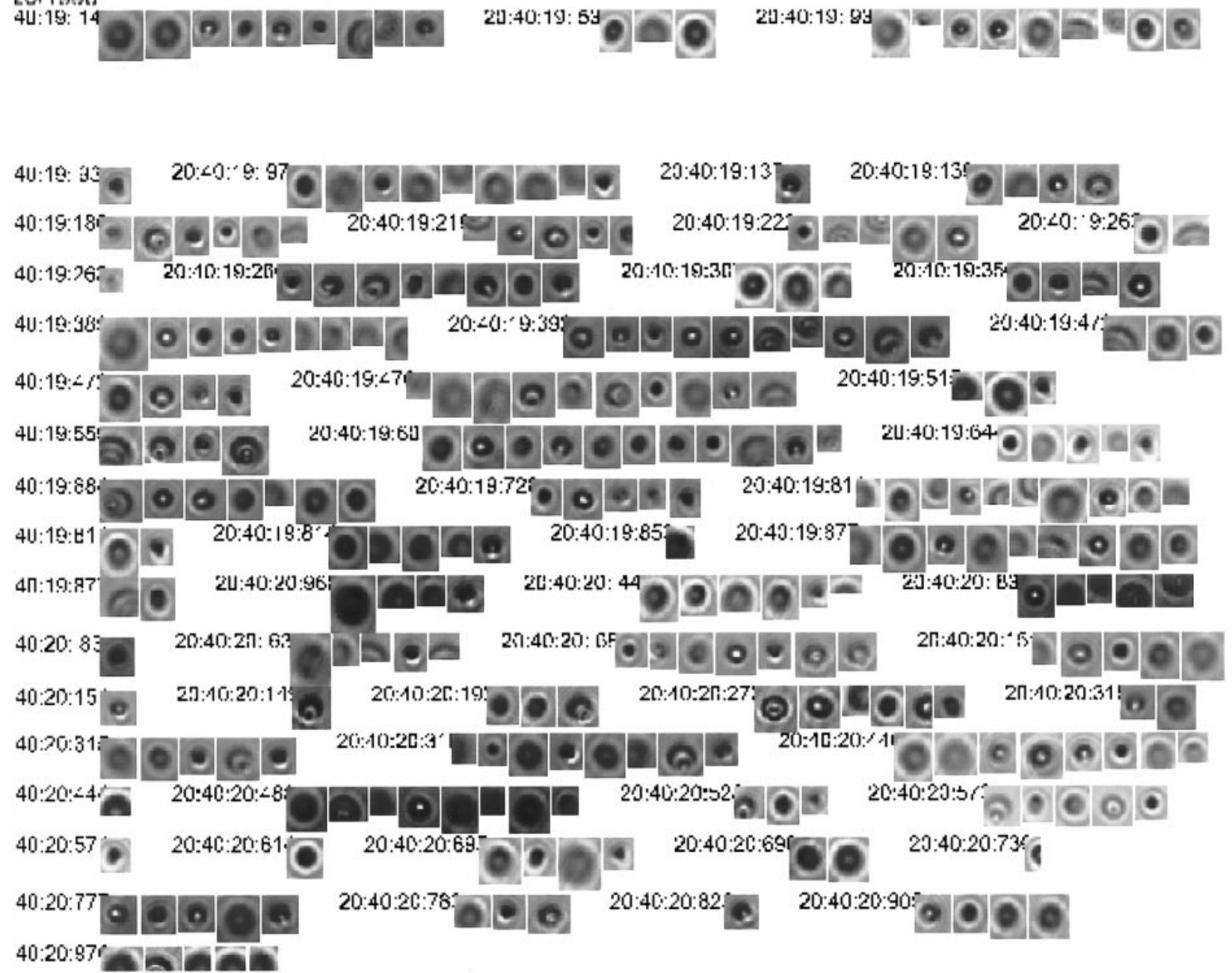

FIG. 9. Images of water droplets collected with the CPI (cloud particle imager; Lawson 1997) during a traverse though a cloud on 20 May. The side of the largest squares is $50 \mu \mathrm{m}$.

was operating as intended. The value of $f$ used here is based on calculations at a wavelength of $550 \mathrm{~nm}$, whereas the CIN operates at $635 \mathrm{~nm}$. This difference in wavelength is not expected to significantly affect the value of $g$.

\section{b. Ice cloud}

Figures 12-14 show 1-Hz measurements made in the glaciated cloud penetrated on 1 June 1998. Typical CPI images for this ice cloud are shown in Fig. 12. They show mostly large multibranched bullet rosettes with some showing as many as 6 or 7 branches. The shape of these rosettes agrees best with the shape of regularaggregate ice crystals modeled by Liou et al. (2000) for which 8 columnar crystals are randomly oriented (No. 13 in Table 1).

The output of the four CIN channels is shown in Fig. 13, which should be compared with Fig. 10 for the water cloud. The comparison shows large differences, in that the ice cloud gives strongly enhanced backscatter in both $B$ and $\cos B$ channels with respect to $F$, and a reduction of $\cos F$ relative to $F$. These results suggest that the ice crystals produce more side scatter than do the droplets in the water cloud, which is consistent with the phase function measurements of Gayet et al. (1998) that also compare the scattering effects of water and ice clouds. The value of $b_{r}$ for the ice cloud is 0.104 , which is about 2.9 times larger than the value of $b_{r}$ for the water cloud. This result differs from the laboratory measurements of Sassen and Liou (1979b), who reported a value for $b_{r}$ of 0.008 for an ice cloud with relatively small crystals, which was about a factor of 0.25 smaller than the value of $b_{r}$ they measured for a water cloud.

The average value of $\bar{g}$ calculated from the measurements of the CIN and Eq. (6) for the 2-min pass throught the ice cloud on 1 June is 0.693 (Fig. 14). This value of $\bar{g}$ corresponds to the value of $f=0.530$ used 


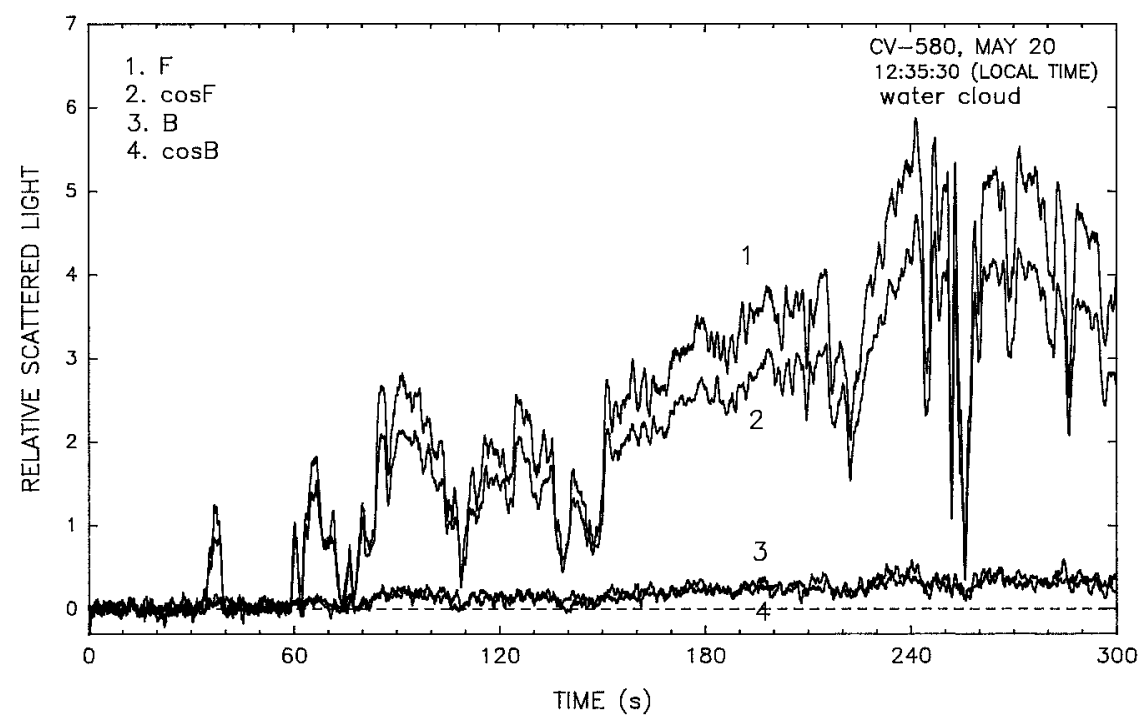

FIG. 10. Relative 1-Hz outputs of the four channels of the CIN during the CV-580 traverse of a portion of the water cloud on 20 May. Forward-scattered light is $F$ (sensor S1); backscattered light is $B$ (S2); forward-scattered and cosine-weighted light is $\cos F(\mathrm{~S} 3)$; and backscattered and cosine-weighted light is $\cos B$ (S4). The data for $F$ and $\cos F$ have been divided by a factor of 5 to permit plotting them on this figure. The local time is h:min:s.

in archiving the data. Table 1 shows that this value of $f$ is too small in comparison to the value of $f(0.5547)$ that corresponds to the regular aggregates (No. 13, Table 1) that have shapes similar to the bullet rosettes found in this ice cloud. A value of $\bar{g}=0.709$ and $\Delta g / \Delta f=$ 0.653 is found by recalculating Eq. (6) using $f=$ 0.5547 . To take into account the expected $\delta$ transmission through these ice crystals, Eq. (8) is used to calculate $\bar{g}_{i}$, given $\bar{g}=0.709$ and the value of $f_{\delta}(0.1001)$ for the regular aggregates listed in Table 1 . This results in $\bar{g}_{i}=0.737$ (standard deviation 0.028), which falls sub- stantially below the mean value of $\bar{g}(0.835)$ for the water cloud as shown by the dashed line in Fig. 14.

Figure 15 shows the relative frequency distribution of the $g$ values measured for the water cloud (Fig. 11) and $g_{i}$ for the ice cloud (Fig. 14). Figure 16 shows the relative frequency distribution of the asymmetry parameter measured in all the clouds penetrated by the CV580 during the first half of its deployment during FIREACE/SHEBA. (In the second half of FIRE-ACE/SHEBA the CIN lost one channel preventing measurements of $g$.) Figure 16 again shows a peak at $g=0.835$, which

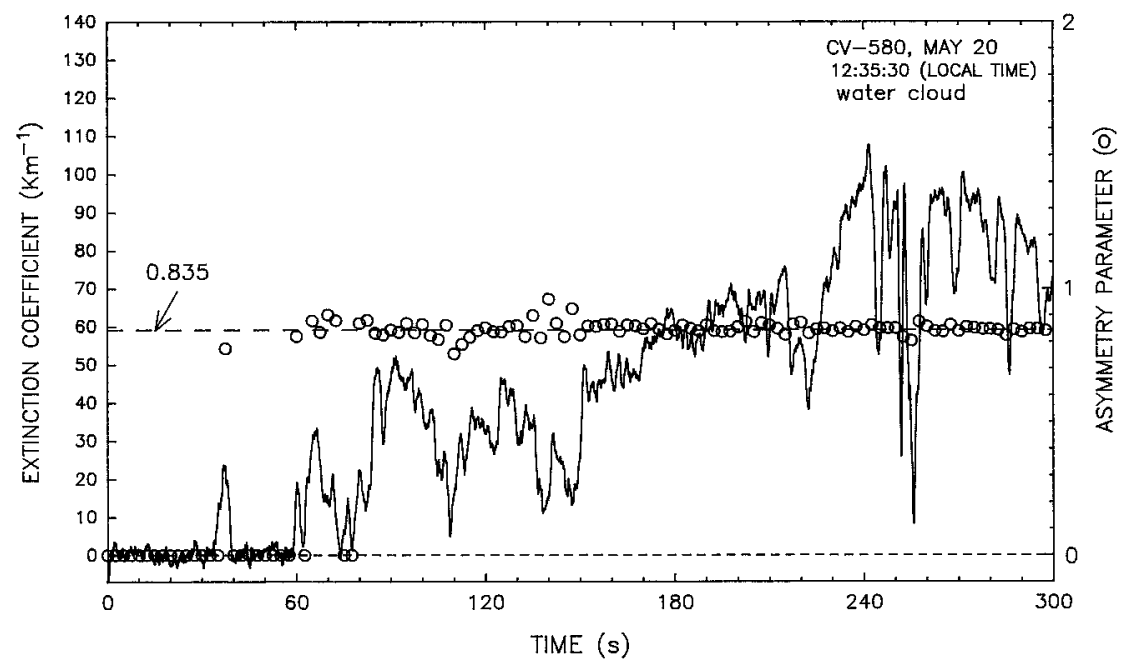

FIG. 11. CIN measurements of the volume extinction coefficient $(\lambda=635 \mathrm{~nm}$ and $g$ in the same cloud as in Fig. 10. The mean value of $\bar{g}=0.835$. 


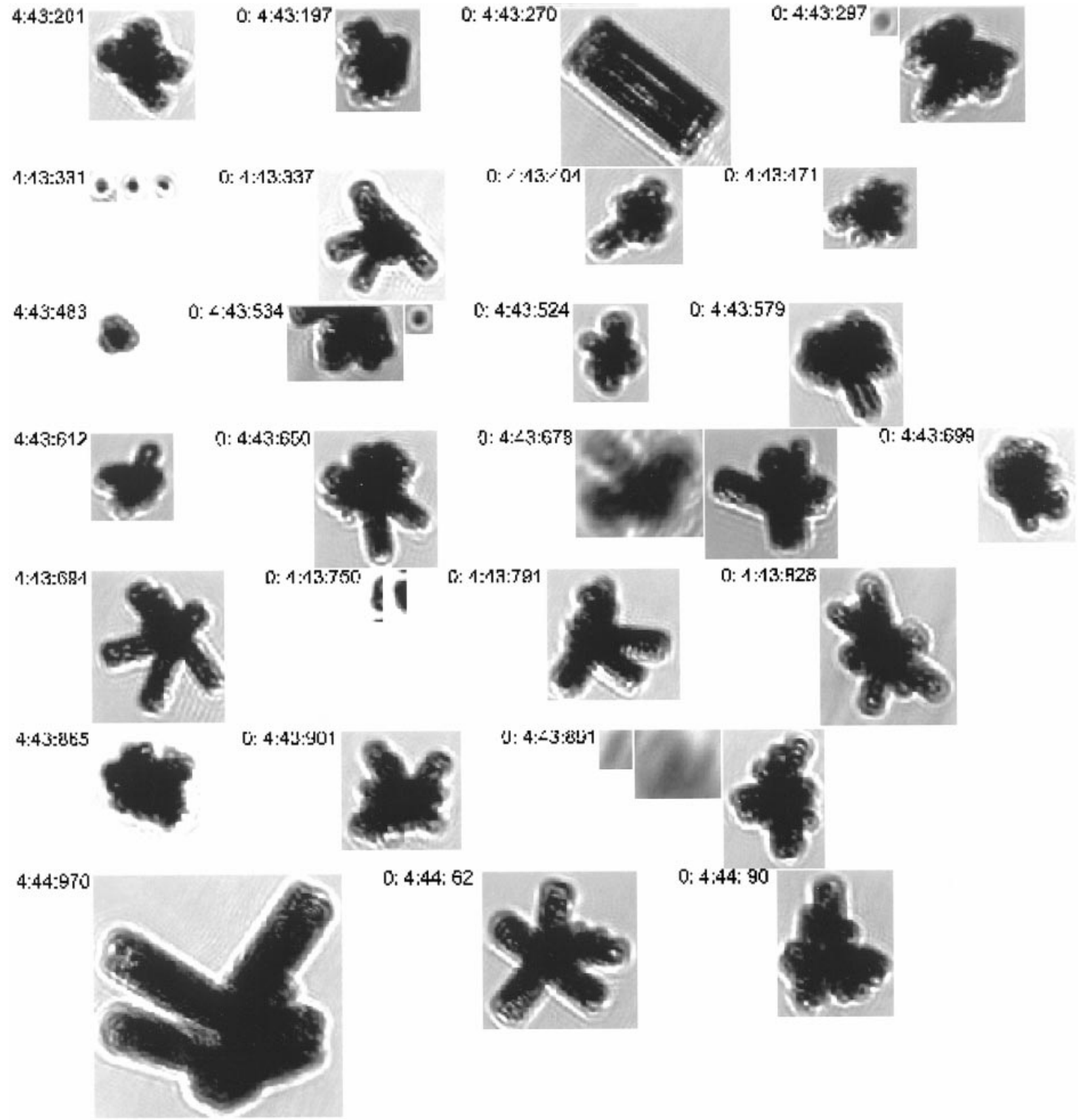

FIG. 12. Images of ice crystals collected with the CPI in a glaciated cloud on 1 Jun. The largest bullet rosette has a maximum diameter of $450 \mu \mathrm{m}$. Figures 9 and 12 are scaled the same.

indicates that water clouds dominated the clouds we sampled. However, the mean value of asymmetry parameter for all clouds has shifted to a slightly smaller value, suggesting a minor effect from ice crystals with smaller values of $g_{i}$.

The spread of the values of $g$ about the mean values in Figs. 15 and 16 is partly caused by random background noise in the signal of all four channels of the CIN. This noise is apparent outside of the clouds (as seen in Figs. 10 and 13). It is not possible to readily separate the background noise from the natural variability of the measured values of $\mathrm{g}$, because the background noise changes in a nonlinear fashion with the amplitude of the CIN output signals. An improvement of the signal-to-noise ratio of the CIN of about a factor of 10 is desirable to reduce the spread of the measured $g$ values, and to permit meaningful measurements to be made in more tenuous ice clouds. 


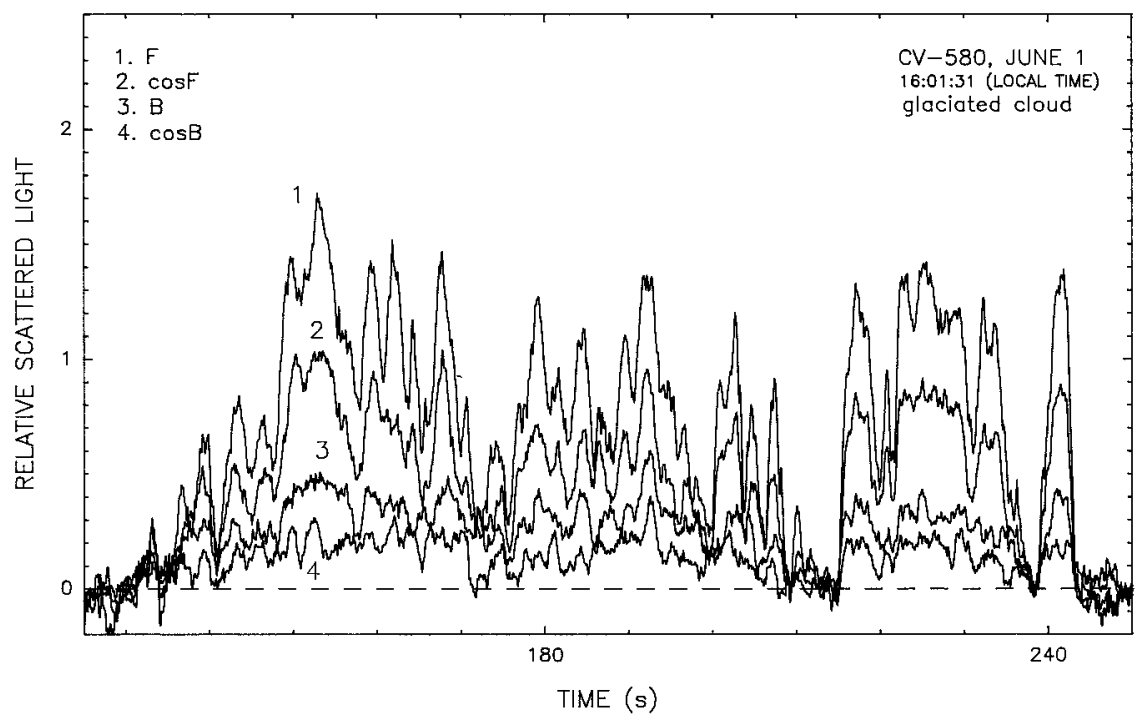

FIG. 13. Relative 1-Hz outputs of the four channels of the CIN during the CV-580 traverse of the glaciated cloud on 1 Jun. The data of $F$ and $\cos F$ have been divided by a factor of 5 to permit their plot on this figure.

\section{c. Discussion}

There is reasonable agreement between the value of $\bar{g}_{i}(0.737)$ measured by the CIN in the ice cloud of 1 June and the value of $g_{i}(0.752)$ for model aggregates (Liou et al. 2000) that resemble the complex bullet rosettes found in this cloud. The remaining difference in the values of $g_{i}$ may be a result of differences between the shapes of the model aggregate ice crystals and the actual ice crystals found in the 1 June cloud, where some of the ice crystals had shapes other than shapes of rosettes as Fig. 12 shows. A second reason for the difference may be the presence of small air-bubble in- clusions in the rosettes, which will tend to lower the modeled value of $g_{i}$ as suggested by Macke and Mishchenko (1999). That the difference between the observed and modeled values of $g_{i}$ is not large suggests that inclusions play a minimal role for these aggregates. The resolution of the CPI images is inadequate to resolve small inclusions or crystal surface irregularities. A final reason for the difference may be CIN instrumentation error.

The value of $\bar{g}_{i}$ found here also agrees well with $g_{i}$ $\approx 0.740$ for the many-faceted and nearly isometric polycrystal model described by Macke et al. (1996), as well

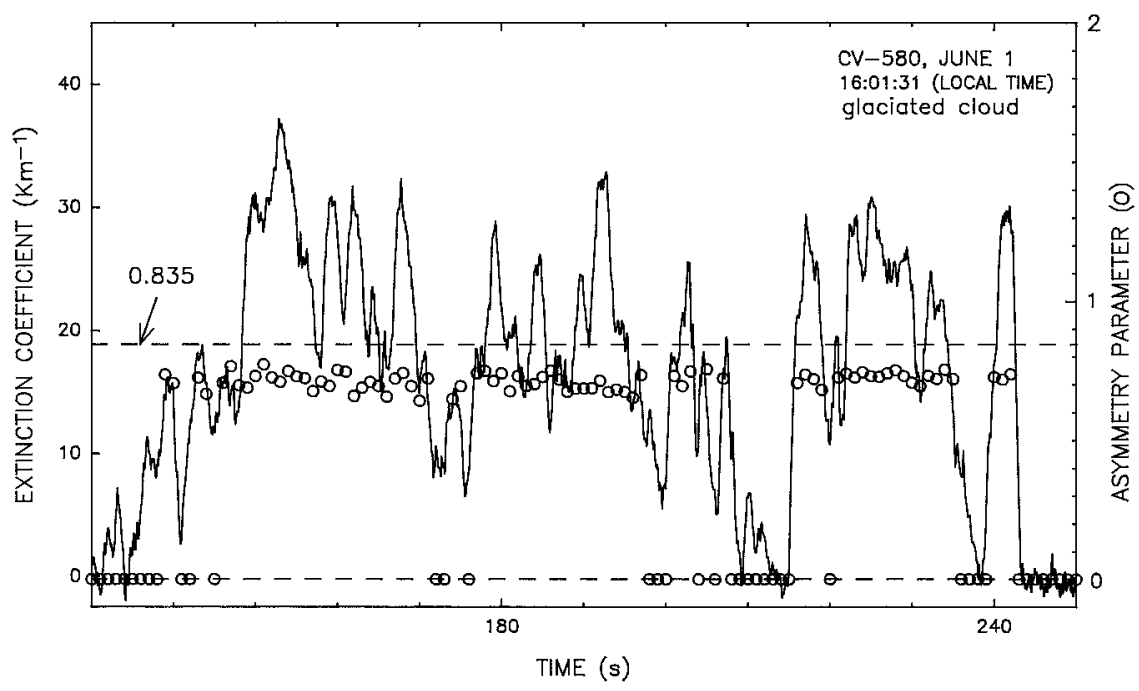

FIG. 14. CIN measurements of the extinction coefficient and $g$ in the same cloud as in Fig. 13. The dashed line labeled 0.835 shows the mean value of $g$ for the water cloud for which data are shown in Fig. 11. 


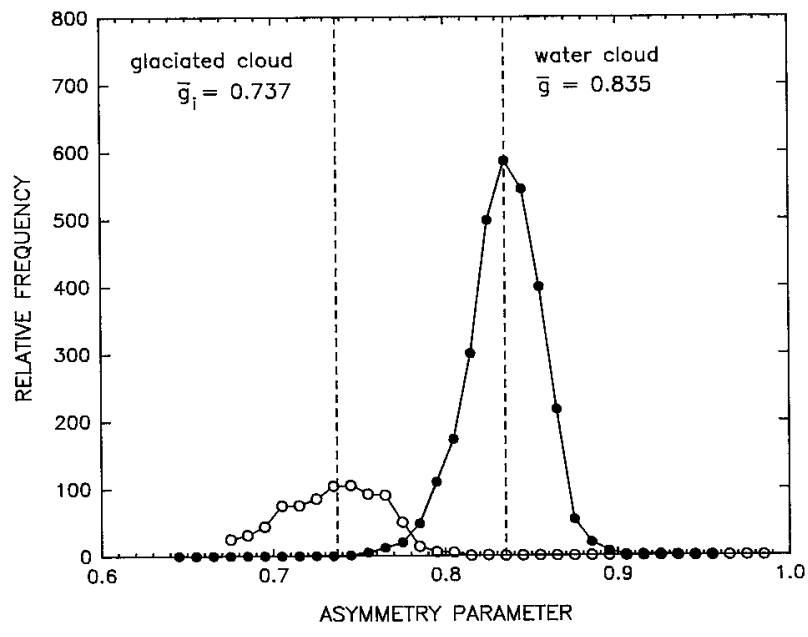

FIG. 15. Frequency distribution of $g$ values measured in the water cloud and the glaciated cloud for which data are shown in Figs. 11 and 14 , respectively.

as with the planar polycrystal model of Mitchell et al. (1996). Macke et al. (1998) give values of $g_{i}>0.800$ for solid hexagonal columns, which are suggested to behave like bullet rosettes. The bullet rosette model described by Takano and Liou (1995) also gives values of $g_{i}$ on the order of 0.800 (Table 1); however, that model decribes rosettes with a much simpler geometry than observed here.

The values of $g_{i}$ calculated from model ice crystals depend in part on the transmission of light through parallel facets of the crystals ( $\delta$ transmission). The effect of this transmission is to increase the value of $g_{i}$ as the fraction of transmitted light to scattered light increases. For example, for the rosettes observed here, $g_{i}$ increases from 0.709 to 0.737 as described earlier. Even greater increases in $g_{i}$ are possible for this effect as illustrated for ice crystals consisting of large and perfect hexagonal plates (Macke et al. 1998). The CIN does not measure $\delta$ transmission, nor have any other such measurements been made on ambient ice crystals. Thus the lack of information on the $\delta$ transmission for real ice crystals increases the uncertainty in the values of $g_{i}$ measured here, as well as in values of $g_{i}$ from model predictions. If ambient ice crystals can be concluded as containing minimal inclusions and as including smooth surfaces, then models can be used to predict the contribution of the $\delta$ transmission to $g_{i}$. The modeled difference in $g_{i}$ with and without the $\delta$ transmission in ice clouds containing a representative selection of different ice crystal types (Takano and Liou 1989, 1995) is not exessive nor highly variable; Table 1 shows that this difference averages about 0.031 for the four listed ice clouds.

Another potential source of uncertainty in the measured values of $g$ is the relationship between the orientation of the CIN on the aircraft and the orientation of ambient ice crystals. This relationship would be unimportant if the crystals can be assumed to be randomly

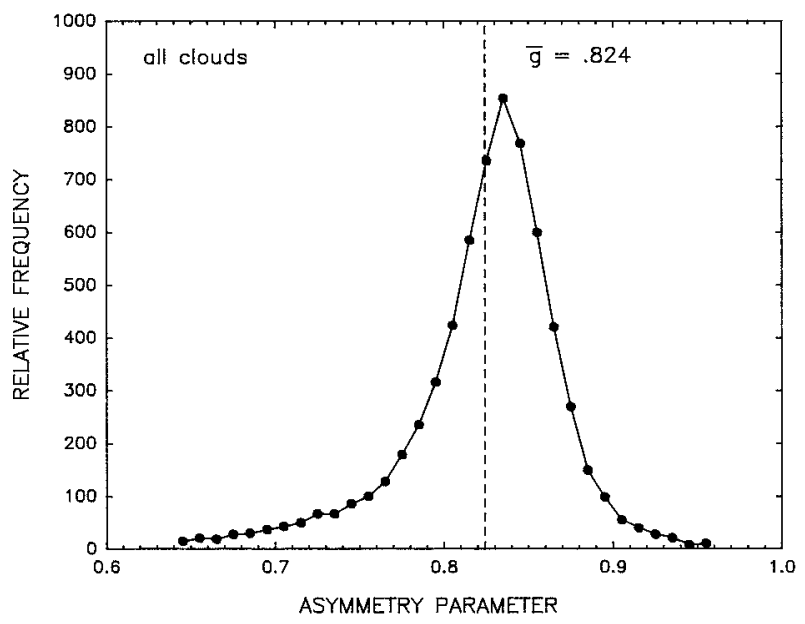

FIG. 16. Frequency distribution of $g$ values measured aboard the University of Washington Convair-580 aircraft for all clouds in the FIRE-ACE/SHEBA field study.

oriented both under ambient conditions and while passing through the instrument. We know, however, that ice crystals can settle in an oriented fashion. For those crystals the measured value of $\mathrm{g}$ would depend on the orientation of the CIN. In addition, nonuniform deceleration of the ambient flow as it approaches the CIN could result in shear that generates torque on the crystals causing them to rotate and change their natural orientation. The importance of these effects is unknown.

Our measured values of $g_{i}$ compare better with the work of Platt et al. (1980), Wielicki et al. (1990), Stephens et al. (1990), and Stackhouse and Stephens (1991), who reported values of $g_{i} \approx 0.700$ from radiance measurements and calculations, than they do with values of $g_{i}$ from more recent efforts by Francis et al. (1994), Francis (1995), Mitchell et al. (1996), and Macke et al. (1998), who gave values $g_{i}$ for ice clouds on the order of 0.800 and larger. If the crystals in our ice cloud are assumed have no $\delta$ transmission, their value of $g_{i}(0.709)$ agrees closely with the earlier values. However, such comparisons are likely meaningless, since we have only measured $g_{i}$ in a cloud with one particular type of ice crystal, whereas the earlier measurements of $g_{i}$ may correspond to clouds with different types of crystals and subsequently different values of $g_{i}$.

\section{Conclusions}

In this paper we have shown that it is feasible to measure the scattering properties $g$ (asymmetry parameter for water drops), $g_{i}$ (asymmetry parameter for ice crystals), $\sigma_{e}$ (volume extinction coefficient), and $b_{r}$ (backscatter ratio) in clouds containing droplets and ice crystals by using the integrating-nephelometer approach. The error caused by the inability of this method to measure scattered light at near-forward scattering angles is corrected by estimating the amount of scattered 
light diffracted in the near-forward direction from measurements of the refracted and reflected scattered light that is spread more evenly over all scattering angles. This permits the nephelometer to integrate the scattered light over a practical range of angles, and to measure the optical properties for droplets and ice crystals larger than about 5 - $\mu \mathrm{m}$ diameter.

The corrections derived for the diffracted light, which are applied to $g, g_{i}, \sigma_{e}$, and $b_{r}$, are based on phase function calculations for a variety of different droplet size distributions, and on phase functions established by a Monte Carlo ray-tracing study for model ice crystals with typical shapes (Takano and Liou 1989, 1995; Liou et al. 2000). The uncertainty in the correction for water droplets is relatively small, and the values of $g$ vary over a small range. The uncertainty in this correction for ice crystals is larger, but still within limits that suggest that meaningful measurements of $g_{i}$ can be made. Measurements with the cloud integrating nephelometer (CIN) of $\bar{g}$ in an Arctic cloud containing only water droplets gave $\bar{g}=0.835$, which compares closely with the exact value of $\bar{g}$ calculated from Mie theory, thus suggesting that the CIN operated as intended during the FIRE-ACE/SHEBA field study.

A source of additional uncertainty in the measured value of $g_{i}$ is the direct transmission of light ( $\delta$ transmission) through parallel facets of the crystals. This unmeasured effect, the importance of which is unclear for actual ice crystals in clouds, can have a significant influence on $g_{i}$, although calculations by Takano and Liou (1989, 1995) and Liou et al. (2000) for ice clouds containing populations of different crystal types suggest an average effect of this transmission that is not excessive nor highly variable, so that it may be possible to take this transmission into account.

The CIN was used for the first time during the FIREACE/SHEBA Arctic field study in May-June 1998. The instrument was deployed on the University of Washington's CV-580 research aircraft. Measurements of $g_{i}$ made by the CIN in ice clouds were of special interest, because there has been no agreement on the value of $g_{i}$, which is an important parameter in radiative transfer calculations. A detailed look at an ice cloud containing mostly large and complex bullett rosettes gave a $\bar{g}_{i}$ value $(0.737)$, which is close to the polycrystal model value of $g \approx 0.740$ given by Macke et al. (1996) and to the regular-aggregate model value $(0.752)$ described by Liou et al. (2000). The average value of $g_{i}$ measured in all the Arctic clouds was 0.824 , which is slightly smaller than the value of $g_{i}$ characteristic for water clouds.

The present results do not resolve the disagreement found in the literature on the value of $g_{i}$ for ice clouds, because the present results only apply to the single ice cloud with the single type of ice crystal analyzed here. The asymmetry parameter depends strongly on ice crystal shape and size so that values of $g_{i}$ given in the literature may correspond to clouds containing different types of crystals. However, the present results do show that small values of $g_{i}$ derived by some researchers from indirect sensing methods may be possible. More detailed studies of the FIRE-ACE/SHEBA data, particularily CIN measurements in ice clouds, as well as more atmospheric measurements of $g_{i}$ are needed.

Acknowledgments. Jack Russell (deceased) and Don Spurgeon of the University of Washington are thanked for their work on installing and maintaining the CIN on the aircraft. This work was supported by NASA Grants NAS1-20506 and NAS1-96020 to Gerber Scientific Inc.; NSF Grant ATM97-96277 to the University of California, Los Angeles; NASA Grant NAG-1-2079, NSF Grant OPP-9808163, and Grant PF904717 from FRONTIER/IARC to the University of Washington.

\section{REFERENCES}

Beutell, R. G., and A. W. Brewer, 1949: Instruments for the measurement of the visual range. J. Sci. Instrum., 26, 357-359.

Francis, P. N., 1995: Some aircraft observations of the scattering properties of ice crystals. J. Atmos. Sci., 52, 1142-1154.

- A. Jones, R. W. Saunders, K. P. Shine, A. Slingo, and Z. Sun, 1994: An observational and theoretical study of the radiative properties of cirrus: Some results from ICE '89. Quart. J. Roy. Meteor. Soc., 120, 809-848.

Gayet, J. F., O. Crepal, J. F. Fournol, and S. Oshchepkov, 1997: A new airborne nephelometer for measurements of optical and microphysical cloud properties. Part I: Theoretical design. Ann. Geophys., 15, 451-459.

_ , and Coauthors, 1998: In-situ measurements of the scattering phase function of stratocumulus, contrails and cirrus. Geophys. Res. Lett., 25, 971-974.

Gerber, H., 1982: Simultaneous measurements of aerosol scattering and extinction in a multipass cell. Light Absorption by Aerosol Particles, H. Gerber and E. E. Hindman, Eds., Spectrum Press, Hampton, VA, 231-241.

_ 1991: Direct measurement of suspended particulate volume concentration and far-infrared extinction coefficient with a laserdiffraction instrument. Appl. Opt., 30, 4824-4831.

— 1996a: Measurement of the asymmetry parameter and optical extinction coefficient of ice crystals and water droplets. NASA Langley Research Center Final Rep. NAS1-20506, 23 pp. [Available from NASA Langley Research Center, Hampton, VA 23681]

_ 1996b: Microphysics of marine stratocumulus clouds with two drizzle modes. J. Atmos. Sci., 53, 1649-1662.

Heintzenberg, J., and R. J. Charlson, 1996: Design and applications of the integrating nephelometer: A review. J. Atmos. Oceanic Technol., 13, 987-1000.

Hobbs, P. V., 1974: Ice Physics. Oxford University Press, 837 pp.

Lawson, R. P., 1997: Improved particle measurements in mixed phase clouds and implications on climate modeling. Proc. WMO Workshop on Measurement of Cloud Properties for Forecasts of Weather, Air Quality and Climate, Mexico City, Mexico, World Meteorological Organization, 139-158.

Liou, K. N., 1992: Radiation and Cloud Processes in the Atmosphere: Theory, Observation, and Modeling. Oxford University Press, $487 \mathrm{pp}$.

, Y. Takano, and P. Yang, 2000: Light scattering and radiative transfer in ice crystal clouds: Applications to climate research. Light Scattering by Nonspherical Particles: Theory, Measurements, and Geophysical Applications, M. I. Mishchenko, J. W. Hovenier, and L. D. Travis, Eds., Academic Press, 417-449.

Macke, A., 1993: Scattering of light by polyhedral ice crystal. Appl. Opt., 32, 2780-2788.

, and M. I. Mishchenko, 1999: Monte Carlo calculations of light 
scattering by large particles with multiple internal inclusions. Preprints, Conf. on Light Scattering by Nonspherical Particles: Theory, Measurements, and Applications, New York, NY, Amer. Meteor. Soc., 143-146.

— - J. Mueller, and E. Raschke, 1996: Single scattering properties of atmospheric ice crystals. J. Atmos. Sci., 53, 2813-2825.

— , P. N. Francis, G. M. McFarquhar, and S. Kinne, 1998: The role of ice particle shapes and size distributions in the single scattering properties of cirrus clouds. J. Atmos. Sci., 55, 2874-2883.

Mitchell, D. L., A. Macke, and Y. Liu, 1996: Modeling cirrus clouds. Part II: Treatment of radiative properties. J. Atmos. Sci., 53, 2968-2988.

Platt, C. M. R., D. W. Reynolds, and N. L. Abshire, 1980: Satellite and lidar observations of the albedo, emittance and optical depth of cirrus compared to model calculations. Mon. Wea. Rev., 108, 195-204.

Rabinoff, R. A., and B. M. Herman, 1973: Effect of aerosol size distribution on the accuracy of the integrating nephelometer. $J$. Appl. Meteor., 12, 184-186.

Sassen, K., and K. N. Liou, 1979a: Scattering of polarized laser light by water droplets, mixed-phase and ice crystal clouds. Part I: Angular scattering patterns. J. Atmos. Sci., 36, 838-851.

, and — $1979 \mathrm{~b}$ : Scattering of polarized light by water droplets. Part II: Angular depolarization and multiple-scattering behavior. J. Atmos. Sci., 36, 852-861.
Stackhouse, P. W., Jr., and G. L. Stephens, 1991: A theoretical and observational study of the radiative properties of cirrus: Results from FIRE 1986. J. Atmos. Sci., 48, 2044-2059.

Stephens, G. L., S.-C. Tsay, P. W. Stackhouse, and P. J. Flatau, 1990: The relevance of microphysical and radiative properties of cirrus clouds to climate and climate feedback. J. Atmos. Sci., 47, 17421753.

Takano, Y., and K. Jayaweera, 1985: Scattering phase matrix for hexagonal ice crystals computed from ray optics. Appl. Opt., 24, 3254-3263.

— Part I: Single-scattering and optical properties of hexagonal ice crystals. J. Atmos. Sci., 46, 3-18.

- , and - 1995: Radiative transfer in cirrus clouds. Part III: Light scattering by irregular ice crystals. J. Atmos. Sci., 52, 818837.

Twomey, S., 1977: Atmospheric Aerosols. Elsevier, 302 pp.

Volkovitsky, O. A., L. N. Pavlova, and A. G. Petrushin, 1979: Scattering of light by ice crystals. Atmos. Oceanic Phys., 16, 90102.

Wielicki, B. A., and Coauthors, 1990: The 27-28 October 1986 FIRE IFO cirrus case study: Comparison of radiative transfer theory with observations by satellite and aircraft. Mon. Wea. Rev., 118, 2356-2376. 